\title{
Popliteal Schwannoma Simulating Vascular Tumor: An Uncommon Case
}

\section{Daoudi Samih, Affes Hassene*,Bouaziz Anis, Kamoun Khaled Anis and Jenzri Mourad}

Orthopaedic Surgery Department, Mohamed Kassab Institute of Orthopaedics, Medicine School of Tunis - Tunis El Manar University, Tunisia

*Corresponding Author: AFFES Hassene, Orthopaedic Surgery Department, Mo-hamed Kassab Institute of Orthopaedics, Medicine School of Tunis - Tunis El Manar University, Tunisia.

DOI: $10.31080 /$ ASOR.2020.03.0190
Received: May 30, 2020

Published: June 26, 2020

(c) All rights are reserved by Affes Hassene., et al.

\begin{abstract}
Schwannomas are benign tumors arising from the Schwann cells on the peripheral nerve sheath. This tumor affects specially adults between 20 and 50 years-old. Malignant transformation is rare and uncommon. The most common clinical presentation of sciatic nerve schwannoma is a painful palpable mass with paresthesia. We present the case of a 57 years old man with a schwannoma of the sciatic nerve. The imaging findings were suggestive of the diagnosis. The tumor was excised without neural damage and no neurological deficit was noted postoperatively.
\end{abstract}

Keywords: Peripheral Nerve Sheath Tumor; Schwannoma; Sciatic Nerve; Benign Tumor; Surgery

\section{Abbreviations}

PNST: Peripheral Nerve Sheath Tumors

\section{Introduction}

Schwannoma or neurilemmoma is a rare benign peripheral nerve sheath tumor arising from Schwann cell. The lower limb is the third location after brachial plexus and upper limb [1]. The size and locations of lesions determine the presence and intensity of symptoms. The goal of treatment is complete excision, with a low rates of recurrence. We present a case of sciatic schwannoma in this article.

\section{Case Report}

A 57-year-old man with a 1-year history of right knee pain was referred to our department with no history of trauma or surgery in the affected leg. The patient reported mild pain along the leg. Physical examination revealed a discrete limping, a solid mass on the right popliteal fossa. We noted the absence of edema and Tinel's sign. Neurological examination and arterial pulse of lower extremity were normal.

Calcifications were noted regarding the popliteal area in the Xray (Figure 1).

MRI showed a well-defined expansive mass at the posterior distal third of the thigh $(52 * 48 * 38 \mathrm{~mm})$ with a large contact with
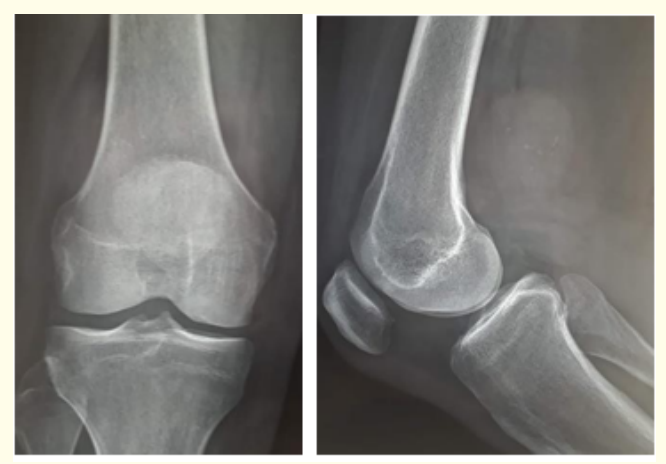

Figure 1: Antero-posterior and Lateral views of the knee showing a mass of the popliteal fossa with calcifications.

popliteal vein and sciatic nerve. This lesion had a low T1 signal, a high heterogeneous T2 signal with moderate uptake of gadolinium (Figure 2 and 3). The compression of the popliteal vein, the presence of calcifications and gadolinium-uptake were suggestive of a vascular tumor.

A CT angiogram of the lower limb showed a well limited mass with centripetal contrast enhancement, probably of venous angiomatous origin with phlebitis (Figure 4).

A surgical resection of the mass by a posterior approach (Figure 5 and 6) with safe margins was decided. 


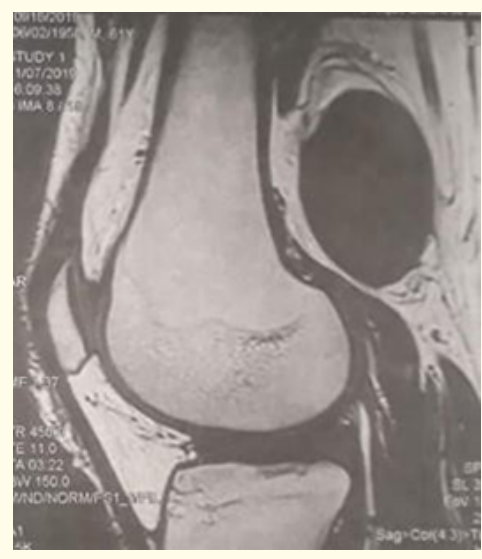

Figure 2: Sagittal T1-weighted image showing a hypo-intense lesion of the popliteal fossa.

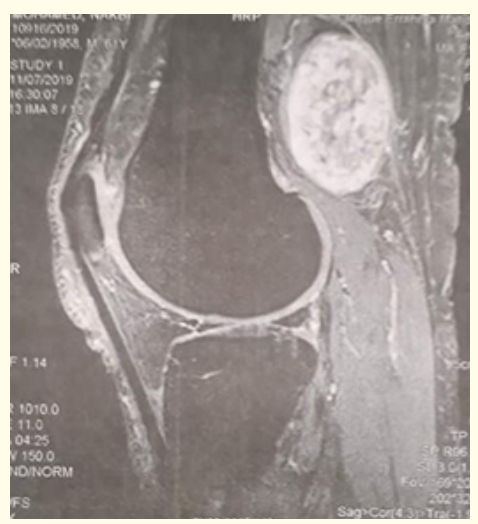

Figure 3: Sagittal T2-weighted image showing a heterogenous lesion of the popliteal fossa.

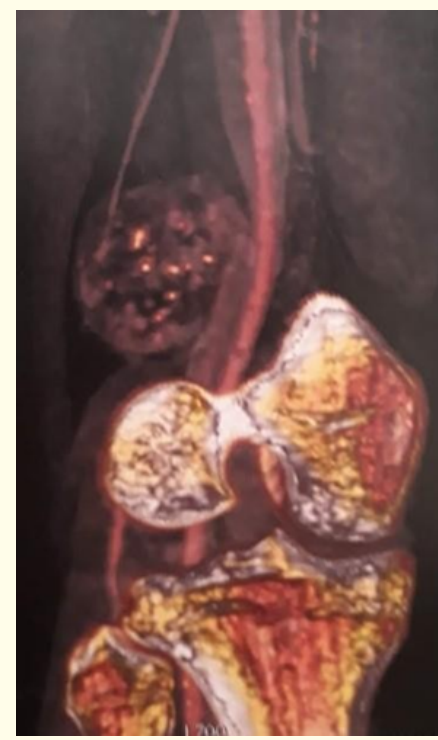

Figure 4: Sagittal T2-weighted image showing a heterogenous lesion of the popliteal fossa.

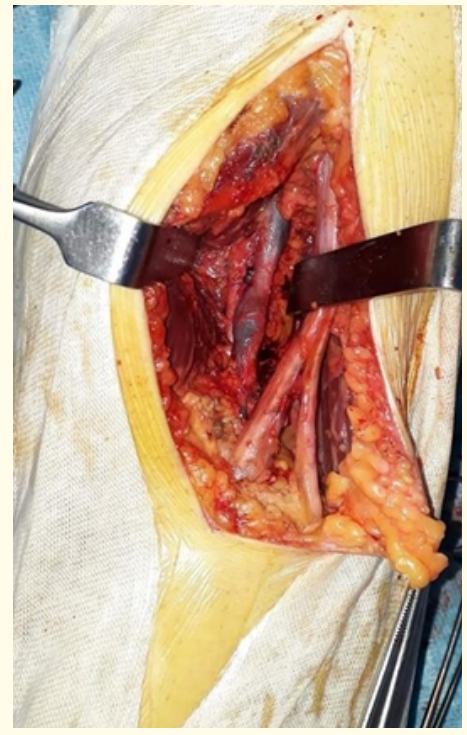

Figure 5: Intra-operative aspect after excising the tumor.

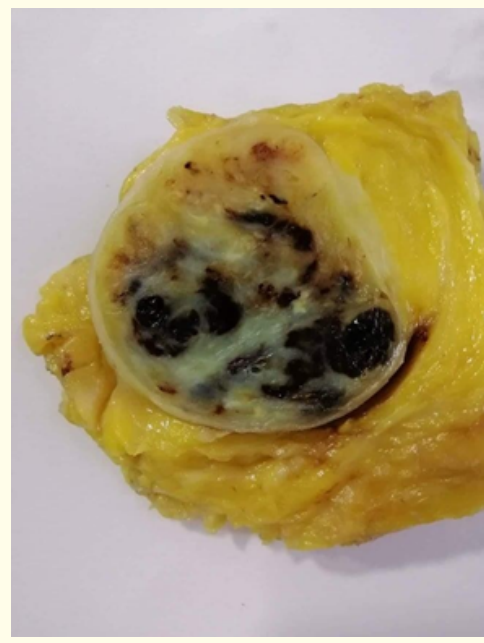

Figure 6: Macroscopic aspect of the specimen.

Histopathological examination of the surgical specimen revealed a schwannoma without signs of malignancy. The immunohistochemical study showed positivity of tumor cells to S100 protein. Post-operatively, the patient showed no signs of any neurological deficit, and the recovery was uneventful.

\section{Discussion}

Schwannoma occurs in six patients per 100,000 [2], with a common age between 20 and 50. No gender predilection was noted in literature [3].

It usually involves upper limbs [4]. The involvement of sciatic nerve is noted in third of cases approximately [1]. Although hereditary disorders especially neurofibromatosis NF1 are associated 
with the occurrence of PNST, the specific etiologies of schwannoma remain unknown. However, an association with previous events of trauma has been suggested in sporadic schwannoma. Our patient didn't report a history of trauma [5,6].

The clinical presentation of schwannoma typically include pain, weakness, numbness and paresthesia. Also, patients present a solitary slow-growing mass. In our case, there was only the pain as a major symptom, but no other specific signs [4].

Any soft tissue mass can cause a compressive effect on the neurovascular pedicle can be a differential diagnosis for schwannoma such as arterial aneurysm, giant cell tumor, tenosynovitis, and ganglion cyst [7].

Radiographs are performed especially to rule out any bone lesion. Ultrasonography can typically show a solid, well- defined and hypoechoic homogenous mass [8,9]. The classic MRI appearance of schwannomas will be a decreased or isointense signal intensity relative to skeletal muscle on $\mathrm{T} 1$ signal and an heterogeneously increased signal intensity on $\mathrm{T} 2$ signal. The presence of the split fat sign, fascicular sign, and target sign are very suggestive of schwannomas $[10,11]$.

The surgical excision is the treatment of choice once the diagnosis has been confirmed in MRI [12]. Sometimes biopsy is mandatory to eliminate malignant peripheral nerve sheath tumours. An electrophysiological stimulation can be used to assess the nerve action potential in the fascicles in the tumor. The NAP tracings are usually flat and the fascicles are sectioned safely [1].

After surgery, paresthesia may be seen but spontaneously resolves; and also recurrence of the tumour is very unusual.

Histopathological examination reveals typically a cellular Antoni A and B areas. Also, immunohistochemical staining (S100) confirms the tumoral cells originating and the diagnosis of schwannoma [3]. This marker was used in our case and the result was positive.

The risk of malignant transformation of peripheral schwannoma is rare [8].

\section{Conclusion}

Neurogenic tumors must be considered when encountering a soft tissue mass. The majority of these tumors are benign. Delay in diagnosis can occur. However, a proper clinical and imaging strategy can lead to the diagnosis.

\section{Conflict of Interest}

No benefits in any form have been received or will be received from a commercial party related directly or indirectly to the subject of this article.

\section{Bibliography}

1. Kim Daniel H., et al. "A series of 397 peripheral neural sheath tumors: 30-year experience at Louisiana State University Health Sciences Center". Journal of Neurosurgery 102.2 (2005): 246-255.

2. Halliday AL., et al. "Benign spinal nerve sheath tumors: their occurrence sporadically and in neurofibromatosis types 1 and 2". Journal of Neurosurgery 74.2 (1991): 248-253.

3. Pilavaki M., et al. "Imaging of peripheral nerve sheath tumors with pathologic correlation: pictorial review". European Journal of Radiology 52.3 (2004): 229-239.

4. Albert Pradeep., et al. "Peripheral Nerve Schwannoma: A Review of Varying Clinical Presentations and Imaging Findings". The Journal of Foot and Ankle Surgery 56.3 (2017): 632-637.

5. Cheng Yi-Shing Lisa., et al. "A forehead mass present for 53 years after trauma in childhood". Journal of Oral Pathology and Medicine 32.10 (2003): 612-617.

6. Wilkinson JM., et al. "Spinal cord schwannoma after vertebral trauma: a causal relation?" Journal of Neurology, Neurosurgery, and Psychiatry 59.4 (1995): 358.

7. Shah A., et al. "A diagnostic approach to popliteal fossa masses". Clinical Radiology 72.4 (2017): 323-337.

8. Jack Christopher Michael., et al. "A case report of three peripheral schwannomas attached to the Achilles paratenon". Foot (Edinburgh, Scotland) 20.2-3 (2010): 78-80.

9. Kwok Ka Bon., et al. "Neurilemmoma of the first branch of the lateral plantar nerve causing tarsal tunnel syndrome". Foot and Ankle Specialist 2.6 (2009): 287-290.

10. Lin J and W Martel. "Cross-sectional imaging of peripheral nerve sheath tumors: characteristic signs on CT, MR imaging, and sonography". AJR: American Journal of Roentgenology 176.1 (2001): 75-82.

11. Beaman Francesca D., et al. "Schwannoma: radiologic-pathologic correlation". Radiographics 24.5 (2004): 1477-1481. 
12. Niepel Albert Laurenz., et al. "Long-term Follow-up of Intracapsular Schwannoma Excision”. Annals of Plastic Surgery 82.3 (2019): 296-298.

\section{Assets from publication with us}

- Prompt Acknowledgement after receiving the article

- Thorough Double blinded peer review

- Rapid Publication

- Issue of Publication Certificate

- High visibility of your Published work

Website: https://www.actascientific.com/

Submit Article: https://www.actascientific.com/submission.php Email us: editor@actascientific.com

Contact us: +919182824667 